

\title{
Metamaterial-Induced TRANSPARENGY Sharp Fano Resonances and Slow Light
}

\author{
Nikitas Papasimakis and Nikolay I. Zheludev
}

\section{Inspired by the study of atomic resonances, researchers have developed a new type of metamaterial. Their work paves the way toward compact delay lines and slow-light devices.}

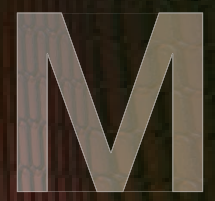

etamaterials-crystalline-like sub-wavelength arrangements of electromagnetic resonatorscan exhibit exotic optical properties, such as negative refraction and cloaking, most of which have no counterpart in natural media. Metamaterial structures could one day be used to develop a wide variety of devices with enhanced and unusual functions, ranging from superlenses to electromagnetic cloaks. Metamaterials are also expected to play a key role in the development of all-optical data processing chips.

Now, a new direction has emerged in metamaterial research. Scientists are looking to realize narrow, low-loss resonances and strongly dispersive behavior in planar, two-dimensional structures of sub-wavelength thickness by using interference effects that suppress radiation leakage. These advances will greatly expand the metamaterial playground to encompass sensors, compact delay lines and coherent light-emitting devices.

At the core of this new approach lies an intriguing analogy with the famous quantum phenomenon of electromagnetically induced transparency (EIT). Following the first observation by Fedotov et al. that the electromagnetic response of certain metamaterials provides "another classical analog of the narrow resonances observed in electromagnetically induced transparency," recent studies show that, by mimicking this quantum phenomenon, the dispersive properties of such planar metamaterials can lead to slow-light propagation and long pulse delays in the microwave, terahertz and optical parts of the spectrum.

Fano resonances: electromagnetically induced transparency and dynamical damping

A typical approach to achieving sharp spectral features involves the so-called Fano resonances that occur due to the interference of different excitation pathways. In the simplest case, Fano interference requires a quasibound state coupled to a continuum - which results in two channels of excitation, a direct one and an indirect one through the quasi-bound state. Constructive and destructive interference of these two channels leads to very narrow resonance lineshapes. Such resonances are used in the well-known phenomenon of EIT, where a pump and probe beam are applied at different dipole transitions of an atom vapor that share the same excited state; transitions between the two ground states are not allowed. 
The resulting destructive interference of quantum probability amplitudes inhibits absorption and leads to a narrow transparency window in the spectrum of the otherwise opaque atomic medium. As a result, the probe beam can propagate without losses. This resonant transmission peak is accompanied by sharp normal dispersion, which can lead to a dramatic reduction in group velocity and a significant enhancement of nonlinear interactions.

However, scientists' observations of EIT in atomic gases were restricted to the available atomic resonances, and the work necessitated optical pumping and often cryogenic temperatures. Such requirements severely hinder practical applications, particularly with respect to integration. These obstacles were soon overcome by the realization that the essential physics behind EIT are actually classical, and similar behavior can be observed in very simple systems, such as coupled spring-mass oscillators.

This insight led to the implementation of induced transparency effects in classical optical systems-for example, coupled optical resonators, photonic bandgap crystals and photonic crystal waveguides - that are robust and do not require special experimental conditions. The operation frequency is directly related to the geometry of the structure and can be varied in a wide spectral range through scaling. Nevertheless, in all approaches to classical EIT, the structure extends along the propagation direction of the incident wave, which imposes restrictions on the minimum dimensions of the medium.

\section{Metamaterial-induced transparency}

In the case of metamaterials, we used Fano resonances in a planar array of asymmetrically split-ring "metamolecules" that consist of two arcs with different lengths in order to minimize scattering losses and achieve highquality resonances. Indeed, breaking the symmetry of the split-ring leads to two closely spaced resonances, each of which corresponds to strong excitation

[ Electromagnetically induced transparency and its mechanical analog ]

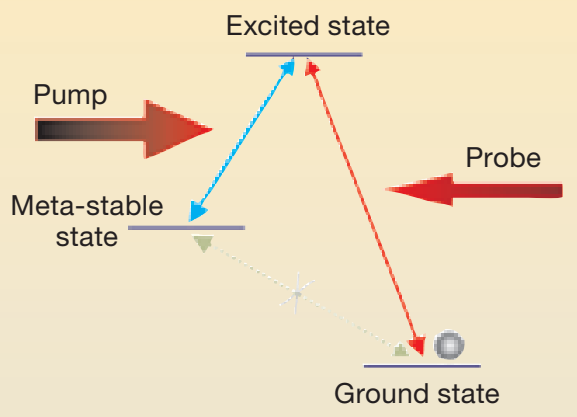

Typical three-level atomic system used in demonstrations of EIT. The ground state is coupled to the excited state, where absorption occurs via a probe beam. A pump beam couples the metastable state to the excited state, while transitions from the metastable to the ground state are not allowed. Interference between the two transitions leads to a vanishing probability for the atoms to be found in the excited state; consequently, absorption is minimized.

Transparency window
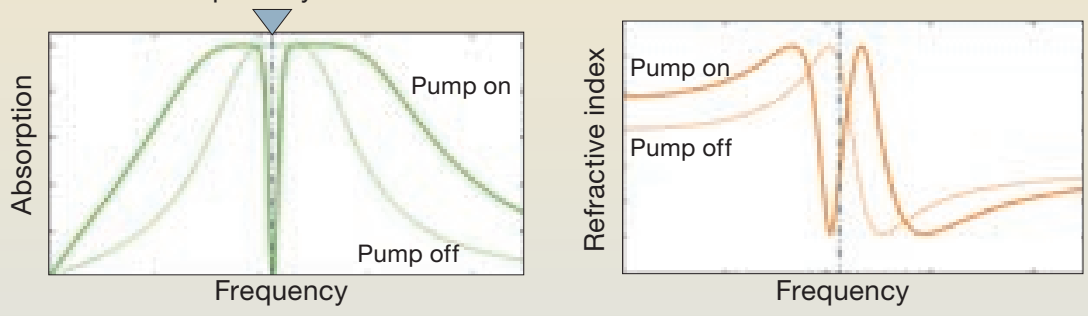

(Left) A characteristic probe absorption spectrum of an atomic medium under EIT conditions. The broad absorption peak experienced by the probe beam is split in two by a narrow dip when the pump is applied. (Right) This results in a very sharp variation of the refractive index, which is responsible for long pulse delays and slow-light behavior.

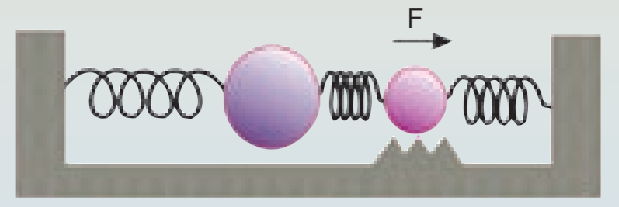

Mechanical analog of an atomic EIT system. The first oscillator is subject to friction and corresponds to the atom under the influence of the probe beam (external force F), while the second stands for the coupling to the pump beam. Under certain conditions, the first oscillator remains still, thus eliminating dissipation in the system.

of one of the two arcs. When excited by an incident electromagnetic wave, the two arcs support currents oscillating in-phase, apart from a narrow frequency range, where an anti-symmetric current configuration is established due to the coupling of the two resonances.

As a consequence, these anti-symmetric currents radiate fields that interfere destructively, allowing the incident wave to propagate without losses, as signified by a narrow transparency window in the transmission spectrum of the metamaterial. This resonant mode has a long lifetime due to its weak coupling to free-space radiation and therefore appears to be "trapped" in the vicinity of the metamaterial surface-hence the term "trapped mode." An important consequence of causality restrictions is that, at the metamaterial resonance, the transmission band is accompanied by steep normal dispersion, providing for low group velocities and slow light behavior.

In fact, we showed in a recent study that similar resonances can also be observed experimentally in a bi-layered structure. In this case, the two metamaterial layers can be either identical or very similar, and they are separated by a small sub-wavelength distance along the propagation direction. This displacement allows the two layers to be excited with opposite phases at a specific 


\section{An important consequence of causality restrictions is that, at the metamaterial resonance, the transmission band is accompanied by steep normal dispersion, providing for low group velocities and slow light behavior.}

frequency, hence leading to the elimination of scattering losses by destructive interference of the re-radiated fields originating from each layer.

After performing an in-depth investigation of the associated dispersive effects, we demonstrated long pulse delays through a thin metamaterial slab by employing classical EIT. The sharp dispersion that occurred at the trappedmode resonance allows for electromagnetic pulses to be significantly delayed with very weak distortion of the pulse shape. Remarkably, this effect is possible in a metamaterial layer with thickness many times smaller than the wavelength of the incident wave-which corresponds to a signal velocity within the metamaterial structure about 200 times smaller than the velocity of light in free-space.

Both in single- and double-layer trapped-mode metamaterials, the width of the resonance (and consequently the pulse delay) can be easily controlled through the geometry of the resonant elements. For instance, increasing the difference in arc length (i.e., the asymmetry) leads to further separation of the individual arc resonances, hence broadening the transparency window of the asymmetrically split-ring metamaterial. On the other hand, the properties of a double-layer structure can be tuned by changing the separation, and thus the coupling, of the two metamaterial layers with longer separation distances, leading to broader resonances and shorter lifetimes.

Intriguingly, the response of EIT metamaterials can be represented by a system of harmonic oscillators. In particular, the oscillating currents on the coupled metamaterial elements can be represented by two harmonic oscillators, with a small difference in their resonant frequencies. The oscillators are coupled through soft springs to a third lighter mass, which accounts for the far-field interference of the two layers.

\section{[ Induced transparency in metamaterials by symmetry breaking]}
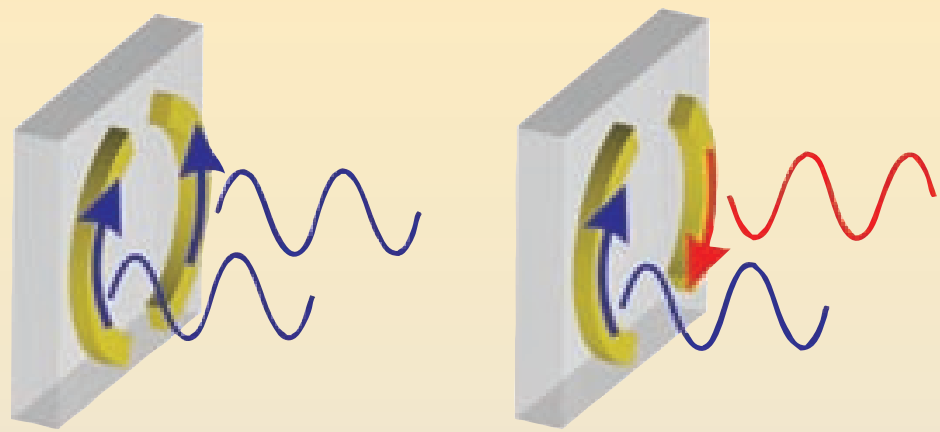

(Left) When the two arcs of the split ring are of equal length, a symmetric current configuration is supported. The scattered waves from the two arcs interfere constructively and give rise to strong scattering. (Right) If the symmetry of the splitring is broken by introducing a difference in the arc length, an anti-symmetric current configuration becomes accessible. The counter-propagating currents radiate fields that interfere destructively, eliminating scattering.
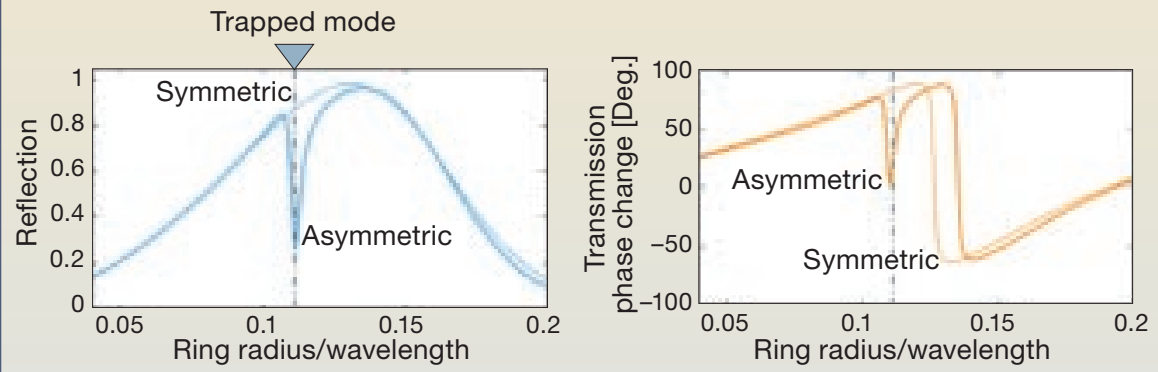

(Left) Suppression of scattering in the asymmetrically split-ring metamaterial leads to a narrow minimum of reflection losses, splitting the symmetric ring reflection peak in two. (Right) At the same time, the phase change of the transmitted wave exhibits steep normal dispersion, leading to low group velocities and long pulse delays.

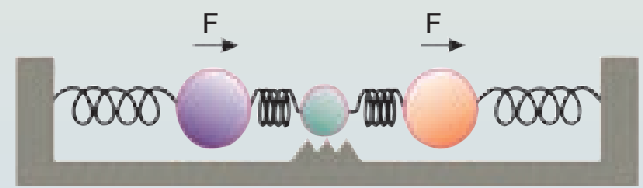

According to a mechanical analog of the asymmetrically split-ring metamaterial suggested by V.A. Fedotov, each arc is represented by a large-mass oscillator coupled to a small-mass, which is subject to friction corresponding to scattering losses. At a narrow frequency range, the large masses oscillate with opposite phases leaving the small mass immobile, hence reducing dissipation.

The small mass is also subject to friction, while the two large-mass oscillators are lossless. The system is excited by an external force acting on both large-mass oscillators, representing the incident electromagnetic wave, while friction stands for the scattering losses in the metamaterial. Normally, the small mass will be forced by the large masses to oscillate, for all driving frequencies, leading to dissipation.

However, the difference in resonance frequencies allows for an antisymmetric 
mode to be established at a specific frequency, where the two large masses oscillate with large amplitudes and opposite phases. As a result, the small mass experiences a zero net force and remains still. All the energy pumped by the external force is stored in the oscillations of the large masses; the dissipative losses in the system are thus minimized.

After the first demonstration of metamaterial EIT, a somewhat different approach was suggested by X. Zhang's research group at Berkeley. Their strategy was based on a "plasmonic molecule" that consisted of a radiative element coupled to a sub-radiant "dark" element. More precisely, the radiative element is strongly coupled to free-space radiation and can be easily excited, while the dark element supports a resonant, perfectly anti-symmetric current configuration, which cannot be excited at normal incidence.

However, it becomes accessible through near-field coupling with the radiative element. In this instance, the two interfering pathways include the direct scattering from the radiative element and the indirect scattering mediated by excitation of the dark element. At a narrow frequency band, energy is efficiently stored in the dark element through its near-field coupling to the radiative element. It is re-radiated again through the latter, leading to strong resonant behavior. The width of the resonance is tuned by changing the distance, and subsequently the coupling strength, of the two elements. Finally, it was shown numerically that a 40 -fold reduction in pulse velocity can be achieved in the suggested plasmonic metamaterial at the visible part of the spectrum.

H. Giessen's group from Stuttgart confirmed experimentally the existence of an EIT-like response in the optical part of the spectrum that consisted of similar plasmonic molecules. The researchers used a meta-molecule that consisted of two coupled split-rings. Following the radiative-dark element approach, one of the split-rings was rotated by 90 degrees, so that when one resonator was strongly excited by a linearly polarized wave, the other remained

[ Metamaterial unit cell for plasmon-mediated induced transparency ]

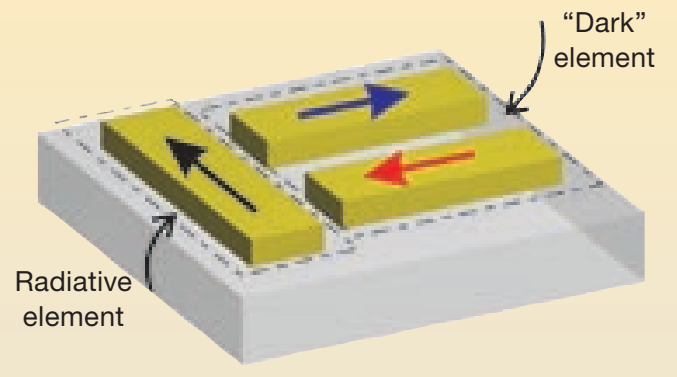

A composite "plasmonic molecule" consisting of a radiative, coupled-to-free-space element and a sub-radiant part. The latter can only be excited through near-field interactions with the radiant element. At a specific wavelength, only the "dark" element is excited, leading to a situation very similar to EIT.

\section{[ Active metamaterial for loss-compensated pulse delays ]}
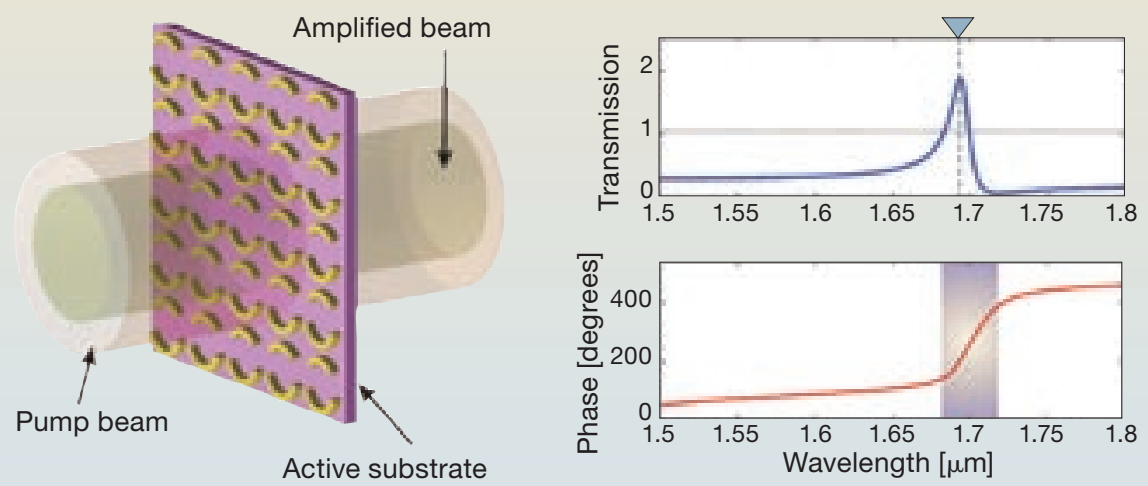

Loss-compensated slow-light device: metamaterial array with EIT-like dispersion placed on a gain substrate $(\varepsilon=9.5+035 \mathrm{i})$. At the wavelength of $1.7 \mu \mathrm{m}$, it shows singlepass amplification and simultaneously sharp normal dispersion.

weakly coupled to free-space. Again, the latter element can be excited by the radiative element through electric or magnetic inductance, leading to a situation very similar to EIT. This analogy was unambiguously demonstrated by the same group very recently, by employing a variant of the meta-molecule suggested by X. Zhang.

Theoretical investigations by P. Tassin and C. Soukoulis' group expanded the concept of EIT metamaterials in the mid-infrared part of the spectrum. Based on a circuit representation of metamaterial resonators, investigators suggested designs for low-loss, strongly dispersive metamaterials. Electric coupling was demonstrated using singlesplit and double-split rings lying on the same plane, while double-split rings lying on perpendicular planes allowed for magnetic coupling. Finally, R. Singh et al. provided further insights into the nature of spectral tunnelling between dark and radiative metamaterial elements in the terahertz domain, where the characteristic EIT behavior was observed experimentally.

Both metamaterial approaches-i.e., trapped-mode and coupled radiativedark elements_-can be considered classical analogs of EIT. Indeed, atomic transitions correspond to the resonances of the meta-molecules, and interference of quantum probability amplitudes translates to classical field interference. In particular, just as the transition amplitudes interfere in the EIT atomic media, the scattered electromagnetic fields from the trapped-mode metamaterial unit 


\section{Metamaterials combine the rigidity, practicality and scalability of other classical approaches with vanishing dimension along the direction of wave propagation.}

cells interfere destructively, resulting in "bright" (strongly radiative) and "dark" (sub-radiant) states. This leads to very similar transmission profiles with asymmetric resonance line shapes that are characteristic of Fano interference controlled by the strength of the coupling of the metamaterial elements.

As in most classical implementations of EIT, the operating frequency of these metamaterials can be tuned using simple geometrical scaling; however, in contrast to all other approaches, the metamaterial structures are planar and do not extend along the propagation direction. In fact, in the microwave and terahertz parts of the spectrum, the structure can have virtually "zero" thickness. On the other hand, in the optical domain, the dimension of the metamaterial along the propagation direction will be limited by the optical skin depth in metals and fabrication restrictions.

In both cases, the thickness of the structures is much smaller than the wavelength, since the resonators lie on the plane perpendicular to the propagation direction of the incident wave. This can lead to very compact realizations of delay lines and possibly components of future optical buffers, where the dimensions are basically limited by the number of resonators required to provide a narrow divergence re-radiated beam.

\section{Addressing limitations}

A number of issues must be addressed before the concept of metamaterialinduced transparency can be transferred to the optical part of the spectrum and find its way to practical applications. The most important limitation-and one that applies to most optical metamaterialsis the strong dissipation associated with metals at these frequencies. This can be overcome by placing a metamaterial array with EIT-like properties on an active substrate, providing moderate gain that leads to the appearance of a narrow resonance of strong single-pass amplification.
Developing this further, we show that a steep normal dispersion occurs at the amplification resonance, thus offering a solution to the loss problem.

For example, we consider an array of silver asymmetrically split rings with a radius of $60 \mathrm{~nm}$ and a cross-section of $30 \times 50 \mathrm{~nm}$. It is placed on a $200-\mathrm{nm}-$ thick active substrate with gain levels that correspond to about 5 percent single-pass amplification of the substrate without the ring array. At the frequency of the trapped-mode resonance, almost two-times single-pass amplification occurs accompanied by very sharp normal dispersion. The required levels of gain can be provided by readily available optically or injection-pumped quantum-well structures or optically pumped semiconductor quantum dots. The feature size of the array is well within the range of the available fabrication capabilities, such as electron beam lithography.

Another notable restriction in the performance of EIT metamaterials is that their operation is limited to normal incidence and specific polarization. However, very recently, we demonstrated an all-angle, all-polarization metamaterial, which is based on concentric rings with slightly different radii. This small difference in ring radius leads to two closely spaced resonances, each of which corresponds to one of the rings. Interference of these resonances leads to EIT-like behavior similar to the asymmetrically split-ring metamaterial. Due to the symmetry of the unit cell resonators, such a metamaterial is isotropic. Weak interactions between different elements in the array minimize the metamaterial angle dependence, rendering it practically insensitive to the angle of incidence for angles up to 45 degrees.

Finally, a common drawback in many approaches is that the resonant sharp dispersion responsible for slow light behavior occurs only within a narrow frequency range. This narrow-band behavior inherent in most strongly dispersive resonant phenomena conflicts with the wide-bandwidth requirements of practical applications, such as optical buffers.

In planar metamaterials, we showed that this restriction can be alleviated, and the bandwidth of operation can be significantly enhanced by the successive stacking of metamaterial slabs. Coupling between adjacent layers leads to multiple closely spaced trapped-mode resonances with continuously normal dispersion. At the same time, the thickness of the resulting multi-layer structure is still much smaller than the wavelength.

\section{In conclusion}

Metamaterials are very attractive candidates for compact optical delay and slowlight devices. They combine the rigidity, practicality and scalability of other classical approaches with vanishing dimension along the direction of wave propagation; this makes them well-suited to manufacturing using existing planar fabrication technologies. $\Delta$

Nikolay Zheludev (niz@orc.soton.ac.uk) and Nikitas Papasimakis are with the OptoelectronOSA ics Research Centre at the University of Member Southampton, United Kingdom.

[ References and Resources ]

>> U. Fano. Phys. Rev. 124, 1866 (1961).

>> S.E. Harris et al. Phys. Rev. Lett. 64, 1107 (1990).

>> C.L.G. Alzar et al. Am. J. Phys. 70, 37 (2002).

> V.A. Fedotov et al. Phys. Rev. Lett. 99, 147401 (2007).

>> N. Liu et al. Adv. Mat. 20, 4521 (2008).

>> N. Papasimakis et al. Phys. Rev. Lett. 101, 253903 (2008).

>> S. Zhang et al. Phys. Rev. Lett. 101, 047401 (2008).

>> N.I. Zheludev et al. Nature Photon. 2, 351 (2008).

>> R. Singh et al. Phys. Rev. B 79, in press (2009).

>> P. Tassin et al. Phys. Rev. Lett. 102, 053901 (2009).

>> N. Papasimakis et.al. Appl. Phys. Lett. 94, 211902 (2009).

>> N. Liu et al. Nat. Mat. 8, 758 (2009). 